\title{
INVESTIGACIÓN GEOFÍSICA COMPLEJA, ANÁLISIS GEOESTADÍSTICO Y MODELACIÓN 3D, CON MÉTODOS ELÉCTRICOS Y NUCLEARES EN MACANAL Y CHINAVITA, BOYACÁ, COLOMBIA.
}

\author{
Fonseca Benítez Fredy Alexander
}

Grupo de Investigación INGEOFISICA; Universidad Pedagógica y Tecnológica de Colombia. Email: frefonseca@yahoo.com

\section{(Recibido: el 3 de Febrero de 2014 y aceptado 14 de Mayo de 2014)}

\begin{abstract}
Resumen:
La implementación de los métodos geofísicos en el departamento de Boyacá es vital en la realización de proyectos que permitan evaluar geológico-geofísicamente las formaciones geológicas y específicamente las unidades litologías presentes en ellas.

A continuación se presenta el marco geológico de la zona de estudio en la cual se realizo la exploración geofísica así como los fundamentos físico - geológicos, la metodología de los trabajos de campo y los resultados obtenidos a partir de la implementación de métodos eléctricos y el método de radiometría.

De igual forma se realizo un análisis geoestadístico de los datos y comparación entre poblaciones por medio de histogramas de frecuencias de los parámetros físicos medidos.
\end{abstract}

Palabras clave: Geoeléctrica, SEV, Radiometría.

\section{Abstract:}

The implementation of geophysical methods is very important in the realization of projects that permit evaluating us the geological formations geologically geophysically and specifically units present lithologies in them.

From now on he shows the survey area's geological frame in which himself I accomplish the geophysical exploration as well as physical - geological basics, the methodology of fieldworks and results obtained as from the implementation of electric methods and the method of radiometry.

Just the same himself I accomplish an analysis geostatics of data and comparison among populations by means of block diagrams of frequencies of physical measured parameters.

Key words: Geoelectric, VES. Radiometry.

\section{INTRODUCCION}

El Departamento de Boyacá presenta gran variedad de litologías pertenecientes a diferentes Formaciones, lo que hace factible un estudio minucioso, con el fin de conocer el comportamiento de la resistividad aparente y la radiación gamma natural.

En la ejecución del proyecto se contó con el equipamiento geofísico y el recurso humano de la Universidad Pedagógica y Tecnológica de Colombia. Se evaluaron los sectores de la zona centro sur del departamento de Boyacá. Se obtuvieron valores representativos de resistividad aparente y la radiación gamma natural en las diferentes litologías presentes a partir de la aplicación de los métodos de Sondeo Eléctrico Vertical (S.E.V.) Perfilaje Eléctrico (P.E.) y Radiometría.

Dicho estudio es de gran importancia en la realización de proyectos posteriores en los cuales se haga necesario conocer el subsuelo y sus respectivas propiedades físico - geológicas y de gran aporte en la caracterización geofísica de las formaciones geológicas presentes en el departamento de Boyacá con el fin de conocer las características geofísicas del departamento lo cual es de gran interés en la aplicación de cualquier ciencia relacionada con la tierra.

Las áreas de estudio se encuentran ubicadas en el flanco este de la cordillera oriental. Los municipios de Macanal y Chinavita se ubican en la zona sur del departamento de Boyacá, en Colombia. Macanal Dista de Tunja la capital del departamento $105 \mathrm{~km}$ y de Bogota la capital colombiana $145 \mathrm{~km}$.
Las zonas de interés se encuentran localizadas entre las coordenadas:

Coordenadas en X: 1088545-1088645 Macanal

Coordenadas en Y: $1042605-1042685$

Coordenadas en X: 1078740-1079160 Chinavita

Coordenadas en Y: 1063070-1042685
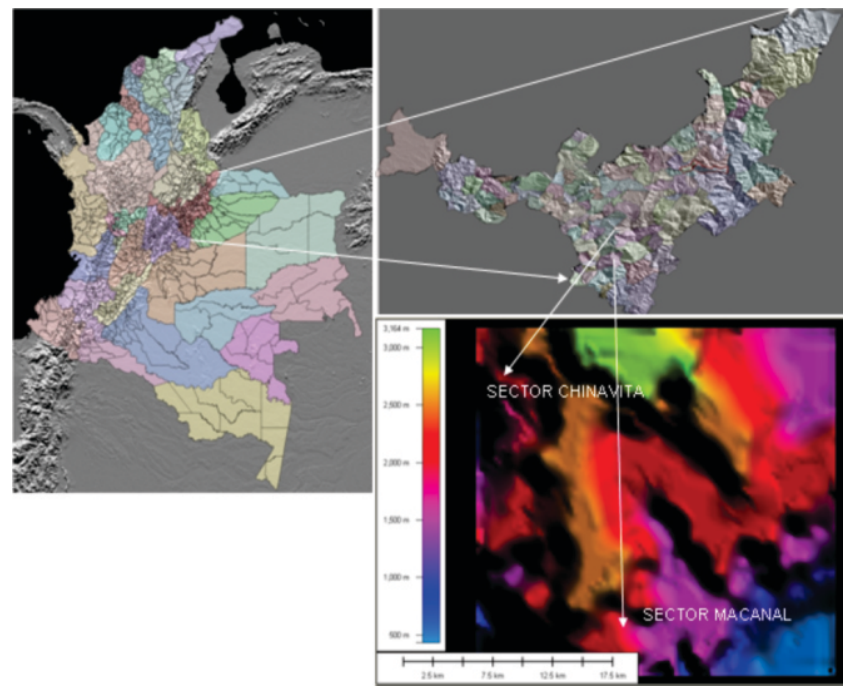

Figura 1. Figura de localización geográfica. 


\section{CARACTERÍSTICAS GEOLÓGICAS}

En general, en la zona de estudio se encuentran rocas del cretáceo y el cuaternario, representada por; Formación Lutitas de Macanal (Kilm), la cual presenta una secuencia monótona de lutitas negras, con intercalaciones de conjuntos de poco espesor de areniscas, calizas y bolsones de yeso; Formación Areniscas de las Juntas (Kiaj), Constituida por una sucesión arenoarcillosa que aflora en los alrededores del caserío de Las Juntas y al NW de la población de Macanal; Formación Fómeque (Kif), compuesta por paquetes de arcillolitas con intercalaciones frecuentes de biomicritas y de algunas arenitas y bioesparitas. La Formación Fómeque aflora ampliamente al NW de Macanal, en los valles de las quebradas Cuya y El Dátil; Formación Une (Kiu), la cual se encuentra constituida por bancos de arenisca intercalados con margas y arcillolitas; Formación Chipaque (Ksc), constituido por esquistos piritosos con intercalaciones de bancos de cal, areniscas calosas, arenisca lajosa y niveles de cal a la base y techo; que tiene gran abundancia de fauna.;

Cuaternario Aluvial (Q), Son aquellos depósitos que se localizan a lo largo de los drenajes del área. Se caracterizan por presentar material no consolidado, arenoso y limoso con escasas barras de gravas; las areniscas son de varias granulometrías.

Tectónicamente se destaca la Fallas inversas de dirección NE inclinadas hacia el NW de la misma forma se presentan estructuras plegadas (Anticlinales y Sinclinales) con dirección paralela a las fallas. Estos plegamientos y la presencia de fallas inversas evidencian la acción de fuerzas compresivas en las rocas presentes en el área y por lo tanto a la cordillera oriental.

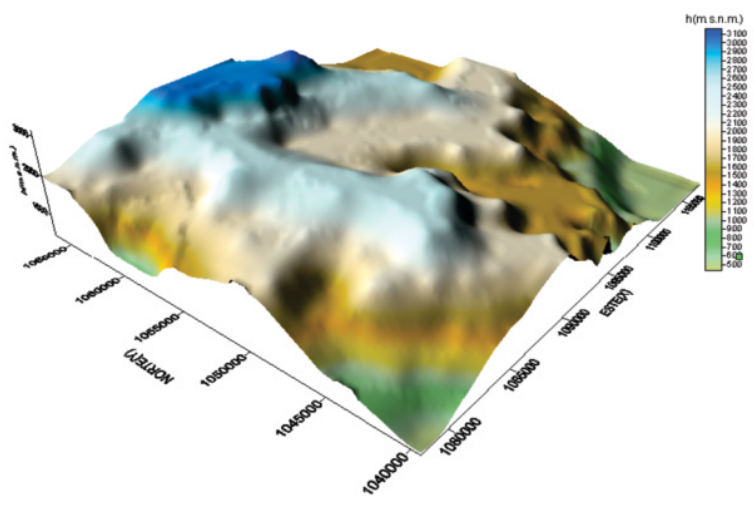

Figura 2. Modelo Digital de Elevación. Sector de Estudio.
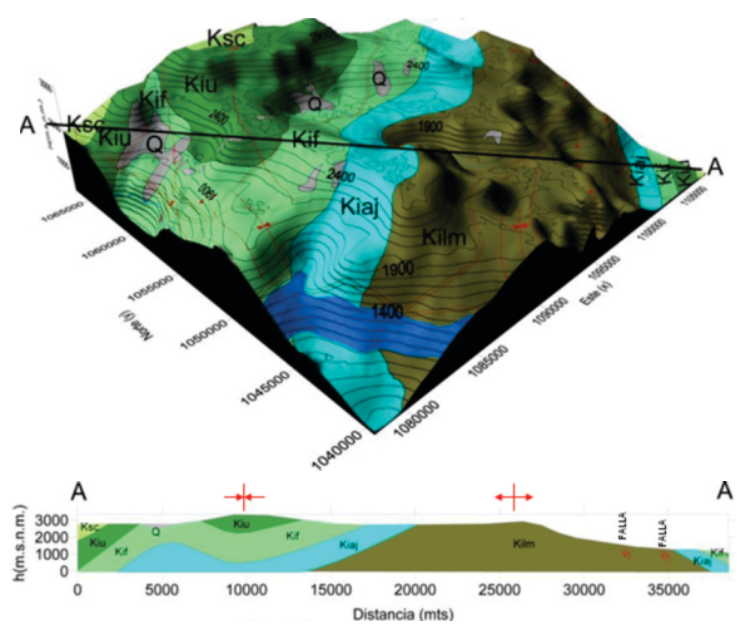

A

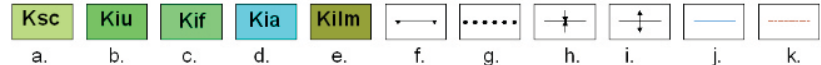

Figura 3. Modelo geológico de superficie y corte geológico dirección NW - SE. Sector de Estudio.

a. Formación Chipaque. b. Formación Une. c. Formación Fómeque. d. Formación Areniscas de las Juntas. e. Formación Lutitas de Macanal. o. y p. Formación Tibasosa. f. Falla de Cabalgamiento. g. Estructura cubierta. h. Sinclinal. i. Anticlinal. j. Ríos y quebradas. k. Carreteras.

\section{MATERIALES Y METODOS}

En la ejecución del proyecto se realizó el siguiente conjunto de actividades:

$\checkmark$ Recopilación Bibliográfica

$\checkmark$ Realización de visitas geológicas de campo con el fin de verificar contactos, ubicación de estructuras y demás información geológica. Elaboración del mapa geológico a escala 1:25000

$\checkmark$ Levantamiento geoeléctrico mediante los métodos de Sondeo Eléctrico Vertical (S.E.V.) y Perfilaje Eléctrico Simétrico (P.E.).

$\checkmark$ Levantamiento con el método de radiometría.

$\checkmark$ Procesamiento representación e interpretación de datos de S.E.V., P.E. y radiometría.

$\checkmark$ Realización de análisis geoestadístico

$\checkmark$ Confección de cortes, mapas y modelos geológicos geofísicos representativos.

$\checkmark$ Elaboración del informe final.

\section{SONDEO ELÉCTRICO VERTICAL}

Los Sondeos Eléctricos Verticales se realizaron con el objetivo de determinar las variaciones de resistividad de los materiales por debajo de los puntos de observación, así como determinar los espesores de esas capas, y evaluar dentro de lo posible, la profundidad del material debajo de la superficie en cada punto (Figura 13, 14, 15 y 16).

Los fundamentos físicos - geológicos del SEV se basan en el estudio de la respuesta del medio geológico al paso de la corriente a través de dos electrodos de alimentación (A y B), que mantienen un circuito cerrado. Este dispositivo fue creado por C. M. Schlumberger. (Figura 4).

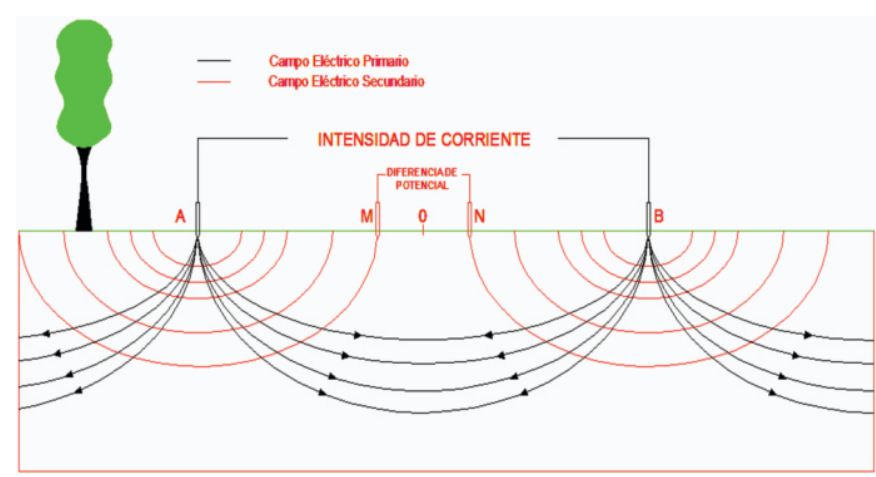

Figura 4. Funcionamiento de la corriente en el dispositivo Schlumberger. (Fuente: FONSECA 2008)
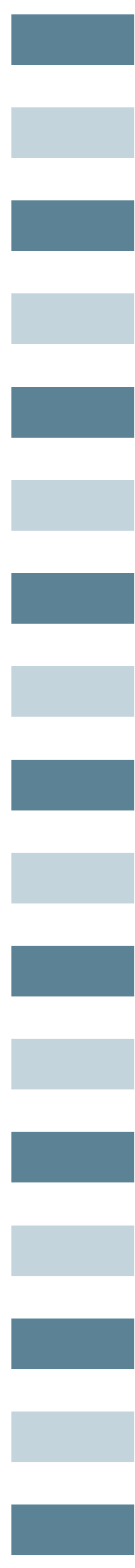$$
\text { Schlumerger, (Fuente: FONSECA 2008) }
$$ 
Los trabajos de campo del SEV consisten en medir repetidas veces en un punto central de observación la diferencia de potencial (U) y la intensidad de corriente (I), para una sola posición de los electrodos de medición, variando la distancia entre los electrodos de alimentación, como se aprecia en la Figura 5 .

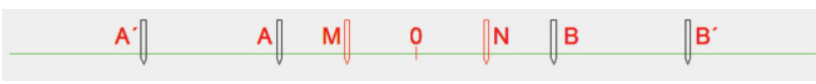

Figura 5. Esquema de ubicación del dispositivo de electrodos de SEV. (Fuente: FONSECA 2008)

Los sondeos eléctricos verticales se localizaron con la ayuda de un GPS, (Global Positioning System). El equipamiento estuvo compuesto por un resistivímetro y un energizador marca PASI, con sus accesorios, según se muestra en la foto 1 .

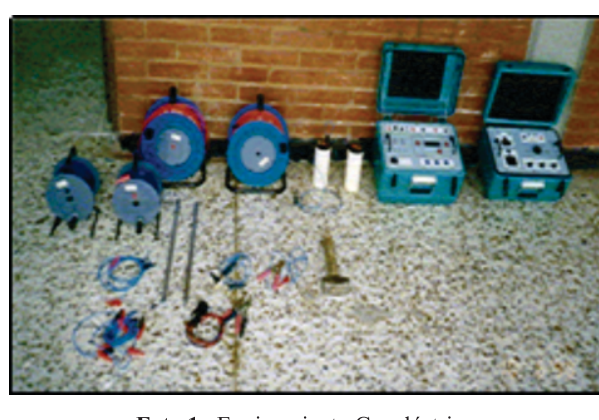

Foto 1. Equipamiento Geoeléctrico.

Se obtuvieron tres datos de diferencia de potencial $\Delta U(\mathrm{mV})$ e intensidad de corriente I (ma) en cada punto de observación. Los datos de campo obtenidos sirvieron para calcular los valores de resistividades aparentes e interpretar las curvas de campo mediante el programa de RESISXP.

Con los tres valores obtenidos, se calculó un promedio de $\Delta \mathrm{U}$ e I, y las resistividades aparentes mediante la fórmula:

$\mathrm{a}=\mathrm{K} \times \mathrm{U}_{\mathrm{MN}} / \mathrm{I}_{\mathrm{AB}}[\mathrm{Ohm} \times \mathrm{m}](1)$

La interpretación de las curvas de SEV y sus respectivos, permitieron confeccionar cortes mapas y modelos geológicosgeofísicos.

\section{PERFILAJE ELÉCTRICO SIMETRICO}

Es un método que como su nombre lo indica, se realiza a lo largo de perfiles, por lo tanto, es un método de investigación horizontal, lo cual permite estudiar la distribución lateral de las resistividades, determinando los contactos verticales e inclinados a diferencia del sondeo eléctrico vertical.

El equipamiento empleado estuvo compuesto por un transmisor, y un receptor que se muestran en la foto 1 .

La metodología de los trabajos de campo se realiza a partir de la observación en varios puntos de los valores de diferencia de potencial y de la intensidad de corriente mediante un dispositivo que mantiene fija la posición de los electrodos de alimentación y de los electrodos de medición, a diferencia del método de sondeo eléctrico vertical (ver Figura 6).

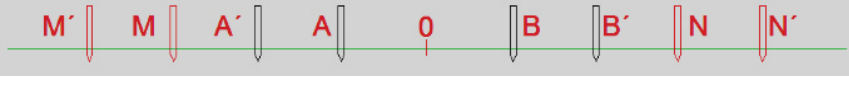

Figura 6. Esquema de ubicación del dispositivo de electrodos de Perfilaje eléctrico Simétrico. (Fuente: FONSECA 2008)

Este es un método que a pesar de ser rápido, tiene el inconveniente de que hay que desplazarse por el terreno con los equipos y transportar todo el sistema de electrodos. A pesar de esto, se considera bastante efectivo para determinar contactos de tipo vertical y sirve como complemento en diferentes estudios geoeléctricos y geológicos.

En el procesamiento se calculan los valores de resistividades aparentes para cada punto del perfil, a partir de la fórmula (1).

Estos valores se plotean en un gráfico en escala normal (milimetrada), donde en el eje Y, se encuentran los valores de resistividad aparente, en ohm $\mathrm{x} \mathrm{m}$, y en el eje $\mathrm{X}$, se encuentran las distancias entre puntos de observación del perfil (estaciones, estacas).

\section{RADIOMETRIAGAMMANATURAL}

Es un método que como su nombre lo indica, mide la radiación gamma natural. En el estudio la información fue obtenida a lo largo de perfiles, por lo tanto, es un método de investigación horizontal, lo cual permite estudiar la distribución lateral de las Intensidades radioactivas gamma, siendo un método de gran utilidad en la determinación de contactos y fallas.

El equipamiento utilizado estuvo compuesto por un Radiómetro SRP 68-02.

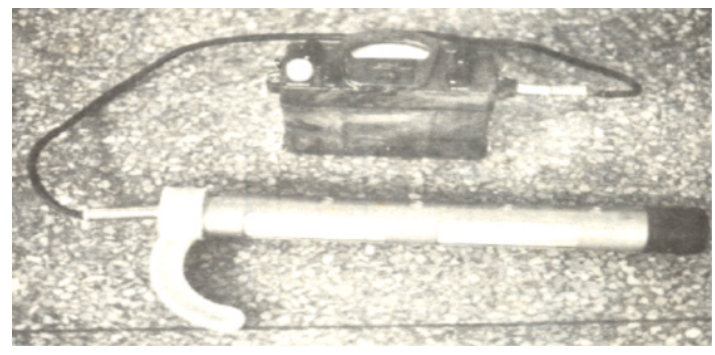

Foto 2. Radiómetro SRP 68-02.

\section{RESULTADOS}

Se exponen dos gráficos de $\rho a\left(\Omega^{*} m\right)$ e ly $(\mu R / h)$ vs. $X(m)$ con sus respectivos cortes geológicos - geofísicos representativos así como dos mapas geológicos geofísicos en cada uno de los sectores, se pueden apreciar las fallas y contactos determinados. Por otra parte se presentan los modelos geofísicos 3D de los parámetros físicos medidos y modelos geológicos geofísicos Superficial $(>1 \mathrm{~m})$ y a $17 \mathrm{~m}$ de profundidad.

Los rangos de resistividades asociados a las litologías presentes en ambos sectores de estudio, se presentan en la Tabla No.1. 
Tabla No 1. Rangos de variación de

resistividades en los sectores de estudio.

\begin{tabular}{|c|c|c|c|}
\hline $\begin{array}{c}\text { Formación } \\
\text { Geológica }\end{array}$ & $\boldsymbol{\rho a}(\boldsymbol{\Omega} * \mathbf{m})$ & $\mathbf{I} \gamma(\boldsymbol{\mu} \mathbf{R} / \mathbf{h}))$ & Litología \\
\hline \multirow{3}{*}{$\begin{array}{c}\text { Lutitas de } \\
\text { Macanal }\end{array}$} & $0-50$ & $>7$ & Yeso \\
\cline { 2 - 4 } & $50-300$ & $3-7$ & Lutitas \\
\cline { 2 - 4 } & $300-1000$ & $1-3$ & Arenitas \\
\hline \multirow{4}{*}{ Une } & $>1000$ & $<1$ & Caliza \\
\cline { 2 - 4 } & $0-50$ & $>10$ & Marga \\
\cline { 2 - 4 } & $150-300$ & $3-10$ & Arcillolita \\
\cline { 2 - 4 } & $>300$ & $1-3$ & $\begin{array}{c}\text { Arenisca } \\
\text { Arcillosa }\end{array}$ \\
\cline { 2 - 4 } & & $1-30$ & $\begin{array}{c}\text { Arenisca } \\
\text { Compacta }\end{array}$ \\
\hline
\end{tabular}

\section{ANALISIS ESTADISTICO}

En el presente trabajo se muestran los histogramas de frecuencias de resistividad aparente e Intensidad gamma natural y se compara por poblaciones además se presenta un grafico tridimensional - multiparametro cual se observan que valores de resistividad Aparente e Intensidad gama se presentan con mayor frecuencia.

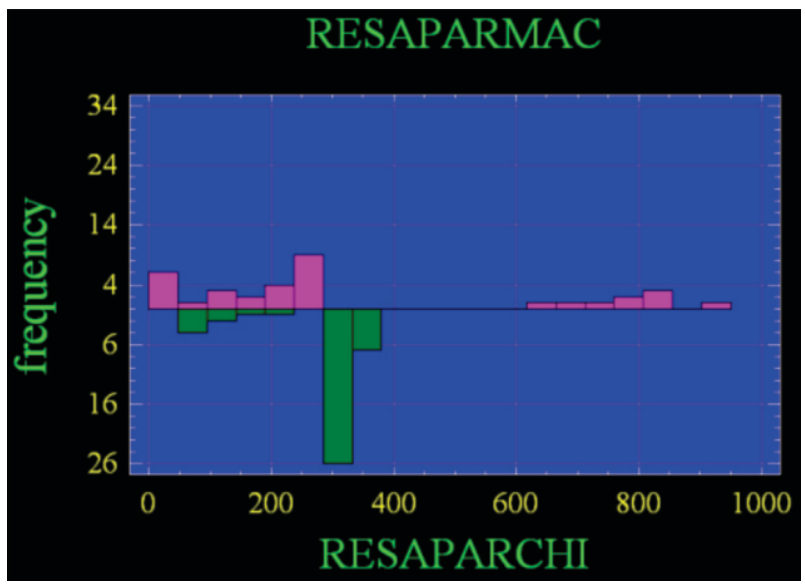

Figura 7. Histogramas de frecuencia vs. Resistividad aparente para los sectores de; macanal (histograma normal) y Chinavita (histograma Invertido)

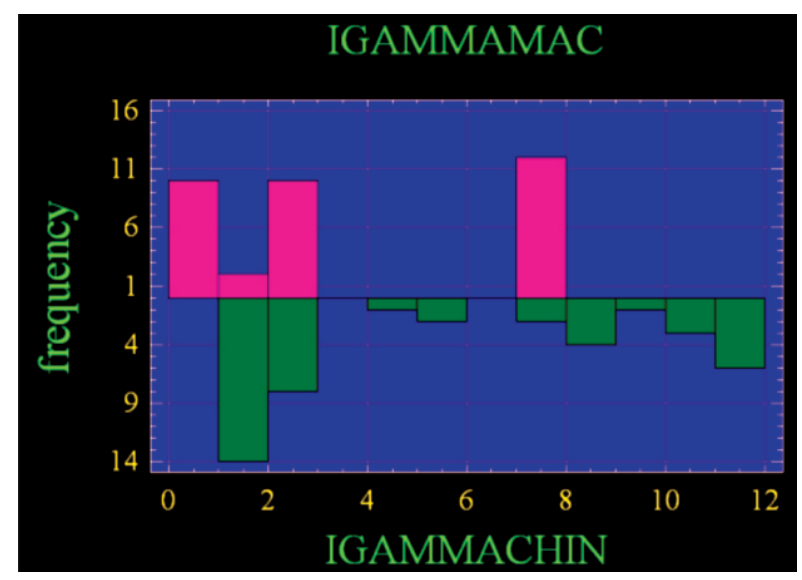

Figura 8. Histogramas de frecuencia vs. Intensidad gamma para los sectores de; macanal (histograma normal) y Chinavita (histograma Invertido)

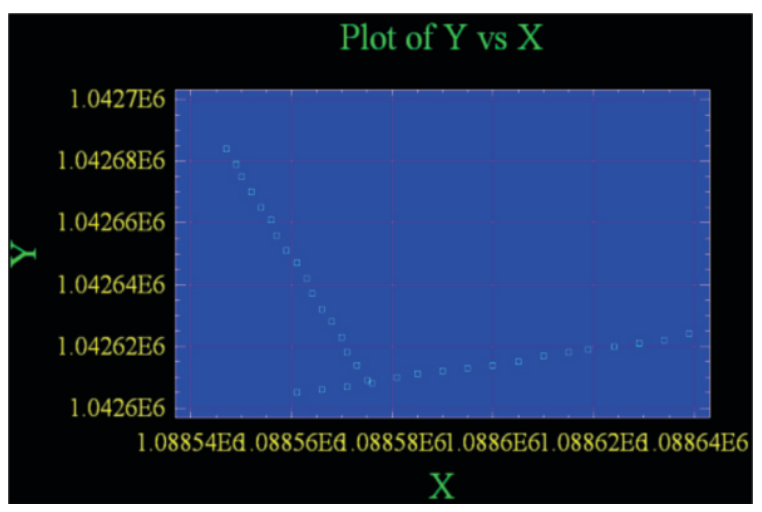

Figura 9. Ubicación de los puntos de muestreo de resistividad Aparente e Intensidad gamma en el sector de Macanal.

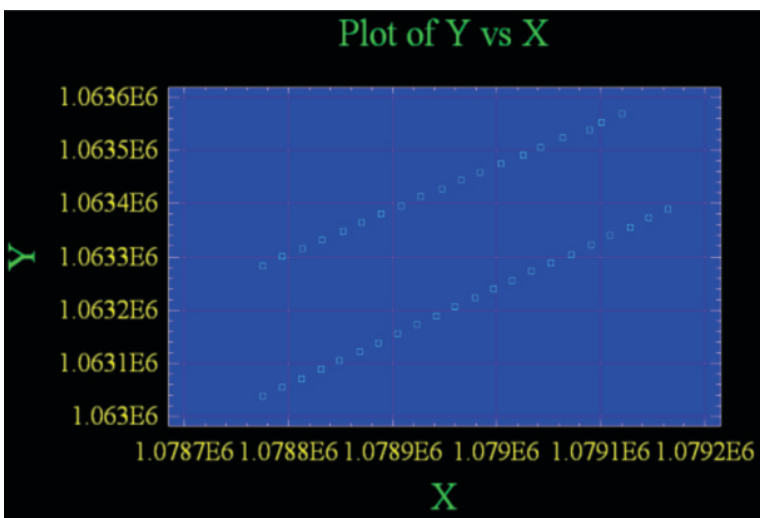

Figura 10. Ubicación de los puntos de muestreo de

resistividad Aparente e Intensidad gamma en el sector de Chinavita.

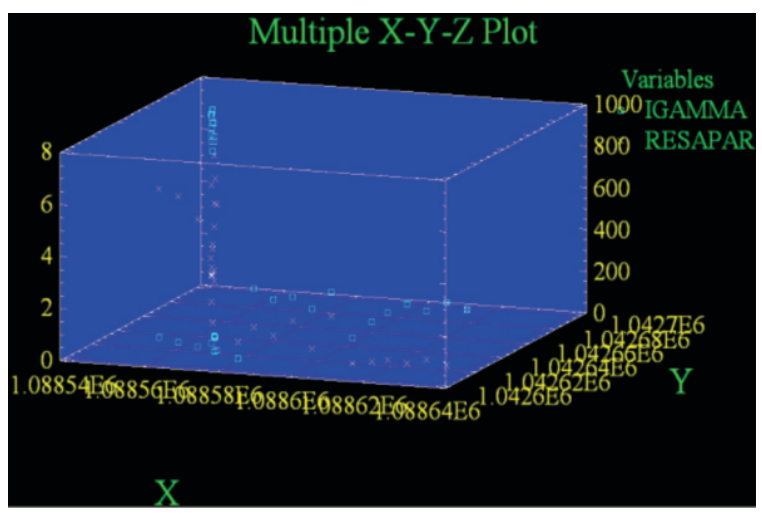

Figura 11. Grafico 3D de los valores de resistividad Aparente e Intensidad gamma en el sector de Macanal

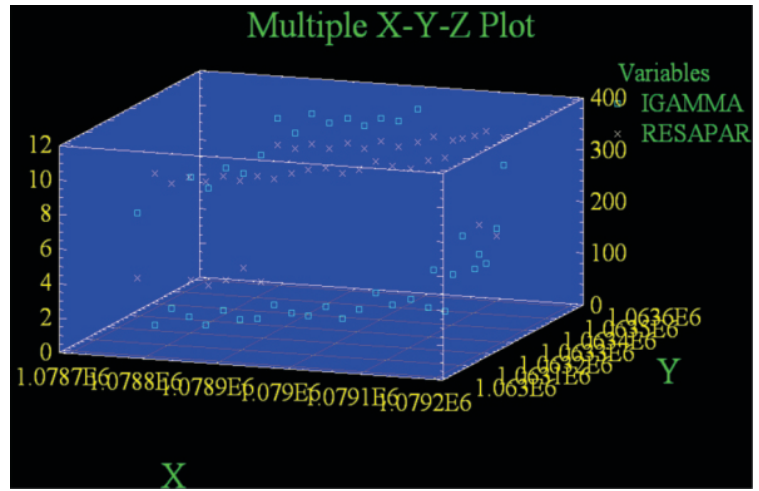

Figura 12. Grafico 3D de los valores de resistividad Aparente e Intensidad gamma en el sector de Chinavita 


\section{SECTOR MACANAL}

\section{CORTE GEOLOGICO GEOFISICO D-D}

Mediante la interpretación geológica - geofísica de los datos obtenidos en campo se logró determinar la posible ubicación de dos fallas locales entre los S.E.V. 6 y 7 (entre la estaca 200 y 250), y los SEV 7 y 4 (entre la estaca 450 y 500). (Figura 13).
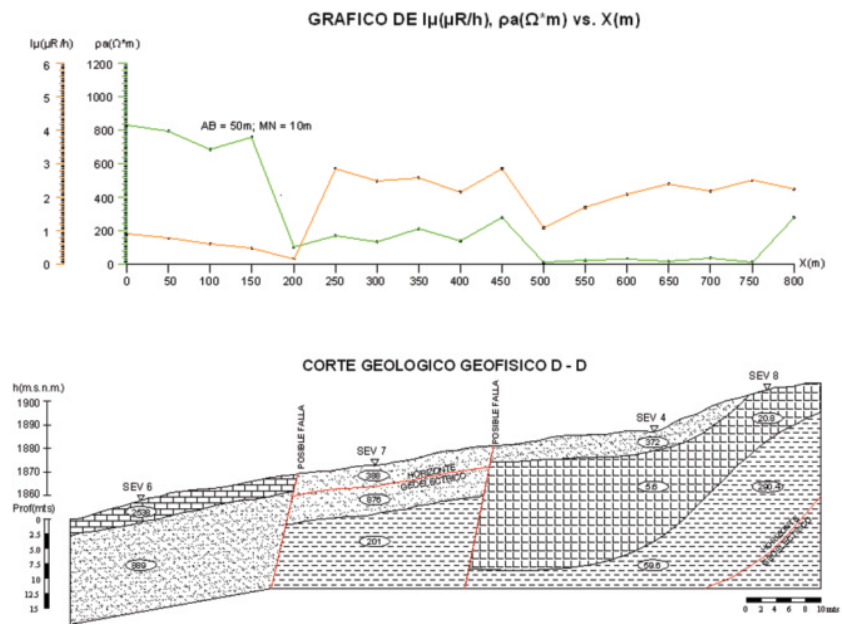

Figura 13. Grafico de resistividad aparente e intensidad gamma y corte Geológico - Geofísico D - D

\section{CORTE GEOLOGICO GEOFISICO E-E}

Con base en la interpretación geológica - geofísica de los datos obtenidos en campo se logró localizar la ubicación posible de la Falla entre los SEV 5 y 9 (entre la estaca 550 y 600 ). (Figura 14)

GRAFICO DE I $\mu(\mu R / h), \rho a\left(\Omega^{*} m\right)$ vs. X(m)
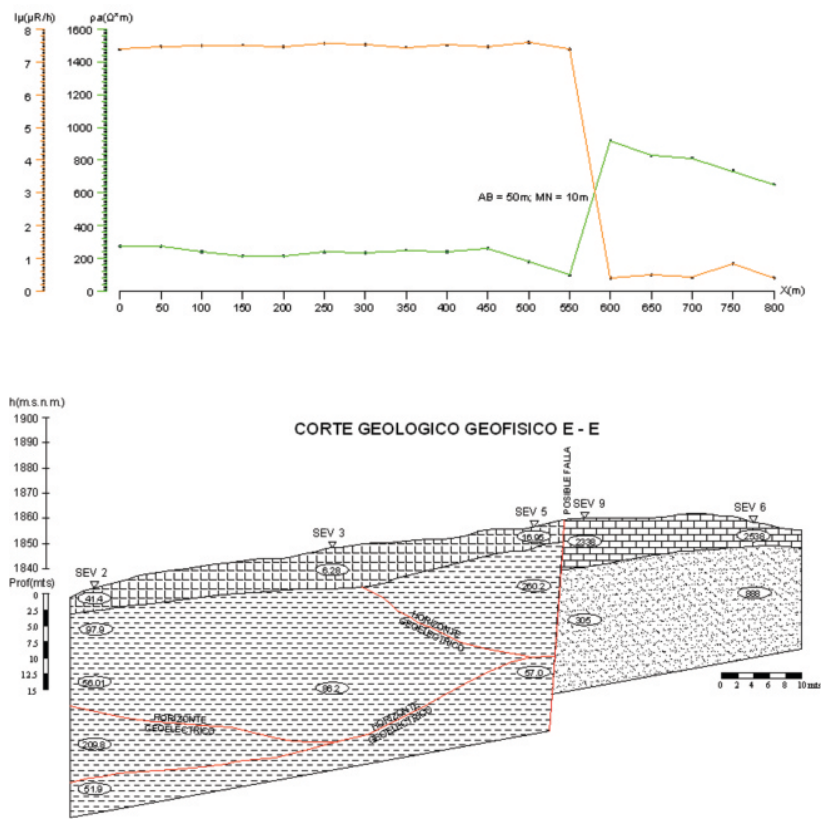

Figura 14. Grafico de resistividad aparente e intensidad gamma y corte Geológico - Geofísico E - E.

\section{SECTOR CHINAVITA}

\section{CORTE GEOLOGICO GEOFISICO F-F}

Mediante la interpretación geológica - geofísica de los datos obtenidos en campo se logró determinar dos posibles Fallas ubicada entre los SEV 8 y 7 (entre la estaca 0 y 250 ) y entre los SEV 5 y 2 (entre la estaca 4500 y 4750). (Figura 15).
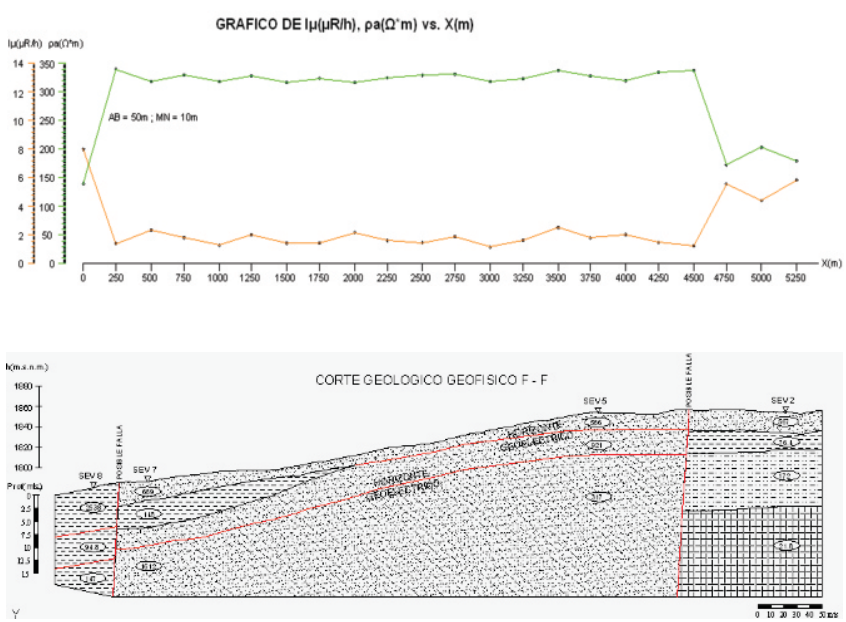

Figura 15. Grafico de resistividad aparente e intensidad gamma y corte Geológico - Geofísico F - F.

\section{CORTE GEOLOGICO GEOFISICO G-G}

Con base en la interpretación geológica - geofísica de los datos obtenidos en campo se logró localizar la ubicación dos posibles Fallas las cuales se ubican entre los SEV 6 y 1 (entre la estaca 1000 y 1250) y entre los SEV 4 y 3 (entre la estaca 4250 y 4500). (Figura 16).

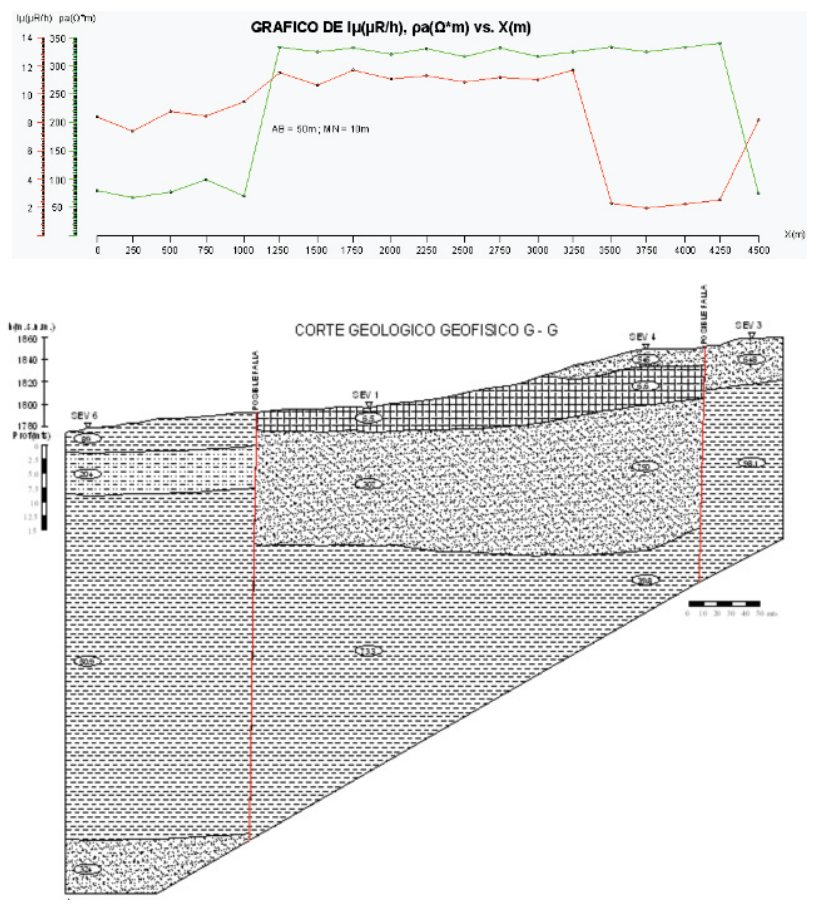

Figura 16. Grafico de resistividad aparente e intensidad gamma y corte Geológico - Geofísico G - G 


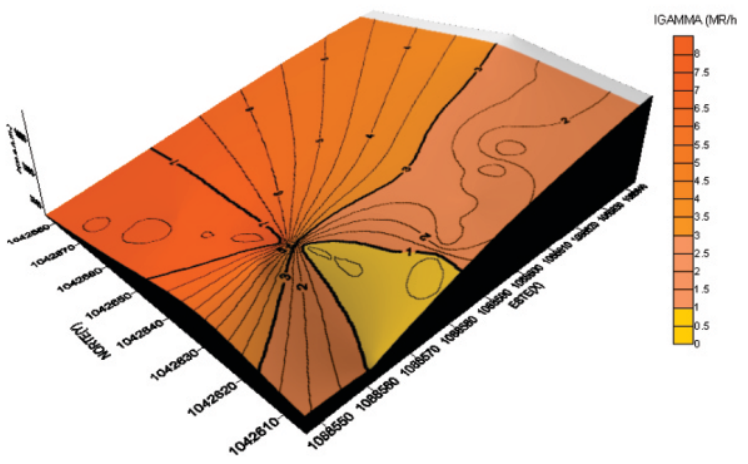

Figura 17. Modelo geofísico de intensidad gamma.

\section{MODELACION GEOFISICAA17 MDE PROFUNDIDAD}

En el modelo se pueden distinguir tres zonas, con mayor frecuencia una zona con resistividad aparente comprendida entre los 50 y los $300 \Omega * \mathrm{~m}$. La zona menos frecuente fue la contemplada entre los valores de 0 y $50 \Omega * \mathrm{~m}$.

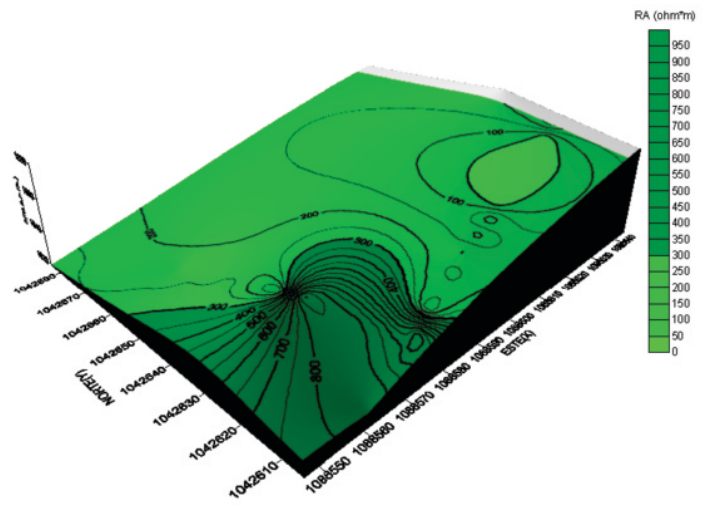

Figura 18. Modelo geofísico de resistividad aparente a 10 s $17 \mathrm{~m}$ de profundidad.

\section{SECTOR CHINAVITA}

\section{MODELACION GEOFISICASUPERFICIAL}

En el modelo se observan cuatro zonas, con mayor frecuencia la zona con intensidad gamma entre 1 a 3 . La zona menos frecuente fue la contemplada entre los valores de 3 y $6 \mu \mathrm{R} / \mathrm{h}$.

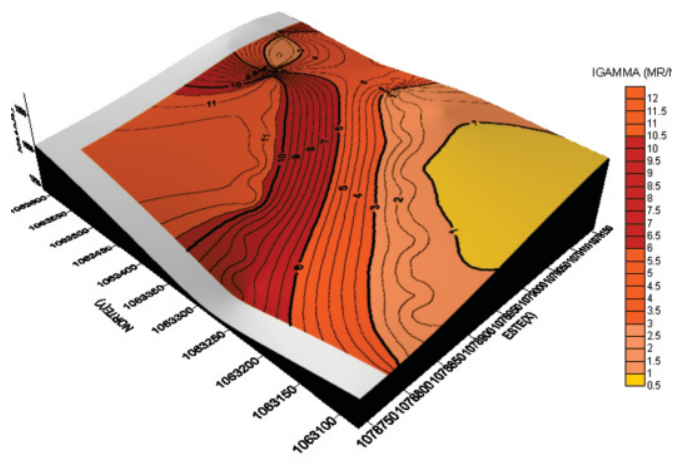

Figura 19. Modelo geofísico de intensidad gamma

\section{MODELACION GEOFISICAA 17 M DE PROFUNDIDAD}

En el modelo se pueden distinguir tres zonas, con mayor frecuencia una zona con resistividad aparente mayor a 300 $\Omega * \mathrm{~m}$. La zona menos frecuente fue la contemplada entre los valores de 50 y $150 \Omega^{*} \mathrm{~m}$.

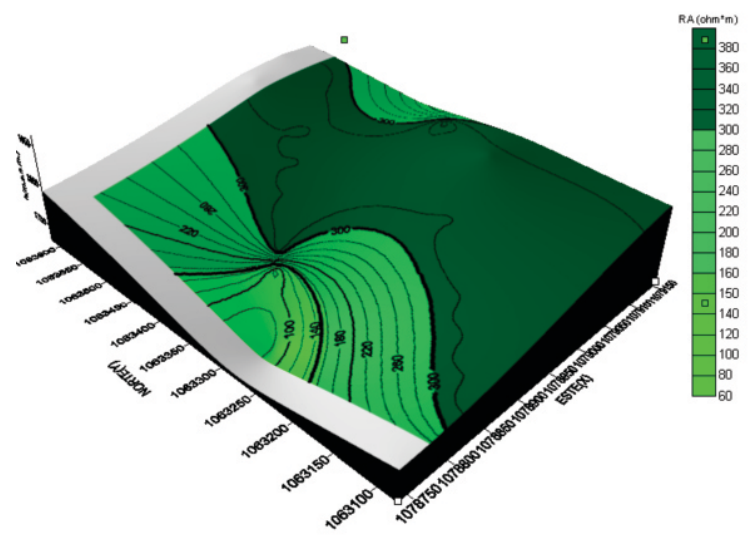

Figura 20. Modelo geofísico de resistividad aparente a los $17 \mathrm{~m}$ de profundidad.

\section{MODELOS GEOLOGICOS-GEOFISICOS FINALES}

Por ultimo se construyeron los modelos geológicos geofísicos finales los cuales muestran la disposición litológica superficialmente y a la profundidad de $17 \mathrm{~m}$.

A continuación se presentan los modelos (Figura 21, 22, 23 y 24)

\section{SECTOR MACANAL}

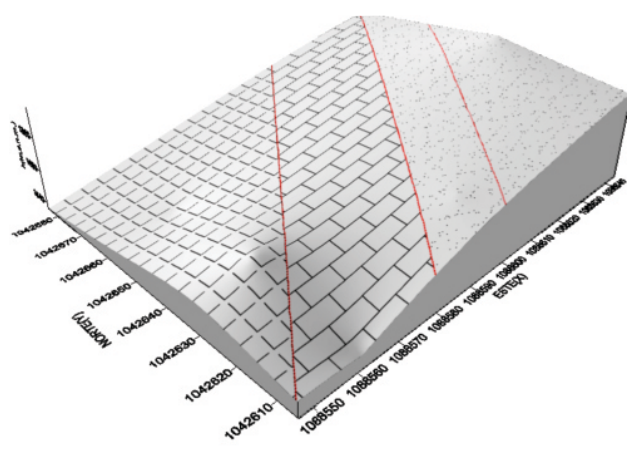

Figura 21. Modelo geológico - geofísico superficial.

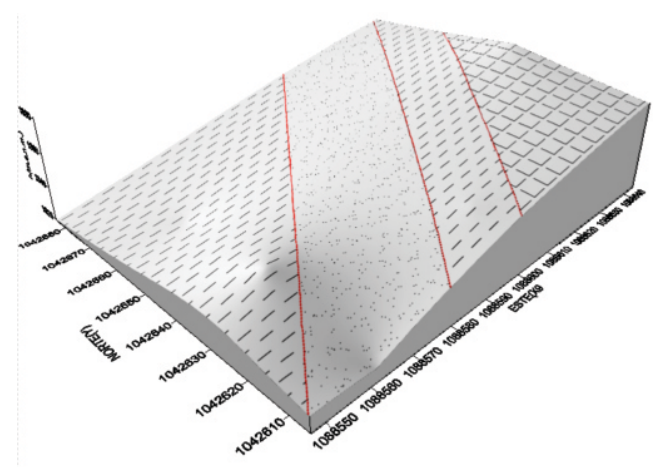

Figura 22. Modelo geológico - geofísico a los $17 \mathrm{~m}$ de profundidad. 


\section{SECTOR CHINAVITA}

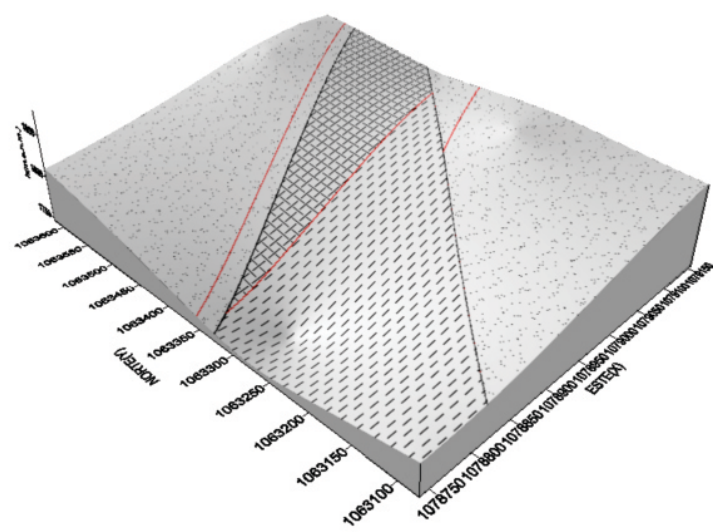

Figura 23. Modelo geológico - geofísico superficial.

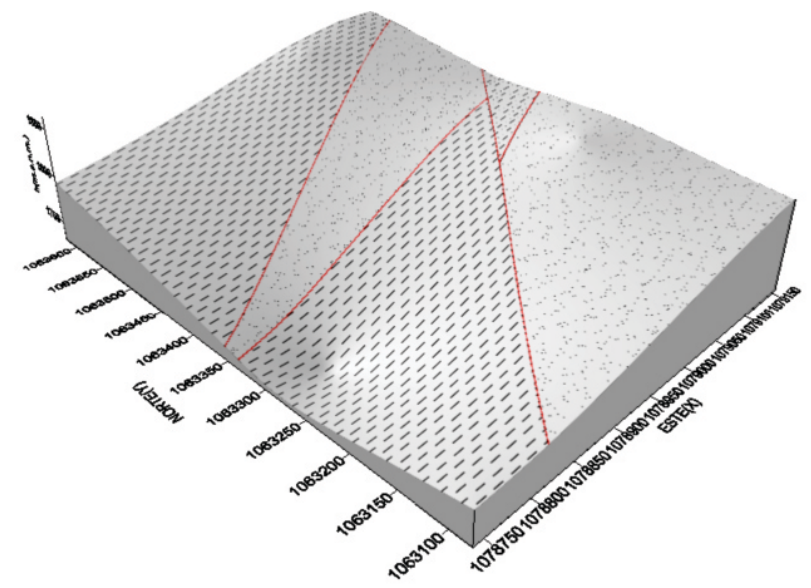

Figura 24. Mapa geológico - geofísico a los 90m de profundidad.

\section{CONCLUSIONES}

La realización de los sondeos eléctricos verticales permitió conocer de manera detallada la geología del subsuelo hasta los $53.3 \mathrm{~m}$.

Al realizar los perfilajes eléctricos y los perfilajes radiometricos gamma en cada área se logró determinar con mayor precisión la ubicación de las zonas de fallas, además de precisar los cambios de resistividades determinados con el SEV.

Se pudo comprobar la efectividad de la aplicación conjunta del método de Perfilaje Eléctrico y radiometría con el Sondeo Eléctrico Vertical.

Se recomienda perforar y correr registros de carácter eléctrico y nuclear

\section{REFERENCIAS}

Fonseca, F. A., (2008). Caracterización geológico geoeléctrica de las litologías presentes en la zona centro de Boyacá. Universidad Pedagógica y Tecnológica de Colombia, Sede Sogamoso. pp. 1-70.

Fuentes, M. C., (2007). Estudios Geoeléctricos en las zonas centro y norte del departamento de Boyacá para el Convenio de Cooperación Acuerdo 015 del 2007, entre INGEOMINAS y la UPTC, Sede Sogamoso, pp. 1-120.

Montoya, D., Y Reyes, G., 2003, Geología de la plancha 209 Zipaquira, Ingeominas, Bogota.

Montoya, D., Y Reyes, G., 2008, Geología del cinturón esmeraldifero oriental Planchas 210, 228 y 229, Ingeominas, Bogota.

Wackernagel, H., 1998, Notas de clases del curso Goeestadística Multivariada, en el Entrenamiento de Postgrados CFSG "Ciclo de Formación de Especialistas en Geoestadística", Curso 1997-98, Centro de Geoestadística de la Escuela Superior de Minas de París, Fontainebleau, Francia. 